\title{
Agroforesterie en développement : parcours comparés du Québec et de la France
}

\author{
par Cécile Tartera ${ }^{1}$, David Rivest ${ }^{2}$, Alain Olivier ${ }^{3}$, Fabien Liagre $^{4}$ et Alain Cogliastro ${ }^{5}$
}

\section{RÉSUMÉ}

La présente analyse établit un parallèle entre l’agroforesterie française et québécoise en fonction de cinq enjeux fondamentaux : reconnaissance, interdisciplinarité et approche collective ; acquisition et transfert de connaissances ; statut, réglementations et financement; sensibilisation et mobilisation; support technique et mise en œuvre sur le terrain. Linformation de base provient notamment d'un forum sur l'agroforesterie réalisé par le Centre de référence en agriculture et agroalimentaire du Québec en mars 2010. Principalement pour des raisons environnementales, les haies et les bandes riveraines arborées tendent à se généraliser; pour leur part, les systèmes agrosylvicoles demeurent moins répandus. Alors qu’en France, la reconnaissance politique est acquise et que de nombreux freins réglementaires ont été levés, au Québec, le statut de larbre hors forêt est encore flou, la réglementation restrictive et les programmes non adaptés. Les systèmes agroforestiers fournissent de multiples services écosystémiques, mais un travail reste à accomplir pour les quantifier et pour établir leur potentiel de production agricole et ligneuse.

Mots clés : agroforesterie, bande riveraine arborée, haie brise-vent, agrosylviculture, culture intercalaire, stratégie de développement, France, Québec

\begin{abstract}
This analysis draws a parallel between France and Quebec agroforestry in terms of five fundamental issues: recognition, interdisciplinarity and collective approach; knowledge acquisition and transfer; status, regulations and funding; awareness and engagement; and technical support and field implementation. For Quebec, the basic information is taken from an agroforestry forum held by the Centre de référence en agriculture et agroalimentaire du Québec in March 2010. Windbreaks and tree riparian buffers are becoming increasingly common, mainly for environmental reasons, while silvoarable systems remain less common. In France political recognition has been achieved and a number of regulatory restrictions have been lifted; however, in Quebec the status of trees outside forests remains unclear, regulations are restrictive and programs are ill suited. Agroforestry systems perform many ecosystem services, but work remains to be done to quantify them and determine their potential in terms of agricultural and timber production.
\end{abstract}

Key words: agroforestry, riparian buffers, windbreaks, silvoarable systems, tree-based intercropping systems, development strategy, France, Quebec

\footnotetext{
${ }^{1}$ Club conseil en agroenvironnement ConseilSol. 740 chemin Trudeau, Saint-Mathieu-de-Beloeil (Québec) J3G 0E2.

${ }^{2}$ Agriculture et Agroalimentaire Canada. 2828 boul. Laurier, local 640, Québec (Québec) G1V 0B9.

${ }^{3}$ Département de phytologie, Université Laval. 2425 rue de l'Agriculture, Québec (Québec) G1V 0A6.

${ }^{4}$ Agroof, 120 Impasse des Quatre Vents, 30140 Anduze, France.

${ }^{5}$ Institut de Recherche en Biologie Végétale, Université de Montréal \& Jardin botanique de Montréal, 4101 est rue Sherbrooke, Montréal

(Québec) H1X 2B2. Auteur correspondant. Courriel : alain.cogliastro@ville.montreal.qc.ca
} 


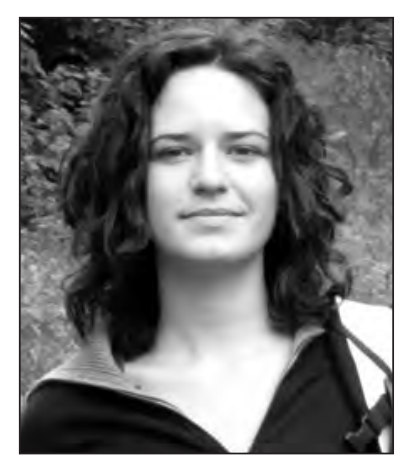

Cécile Tartera

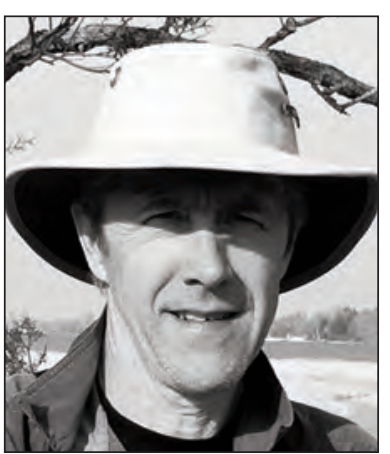

Alain Cogliastro

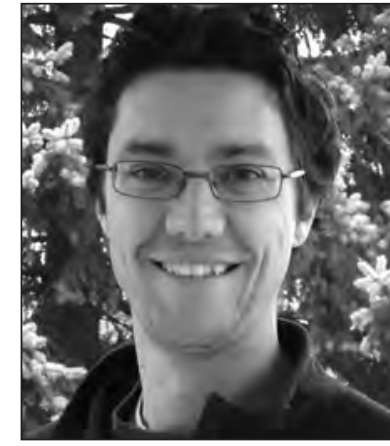

David Rivest

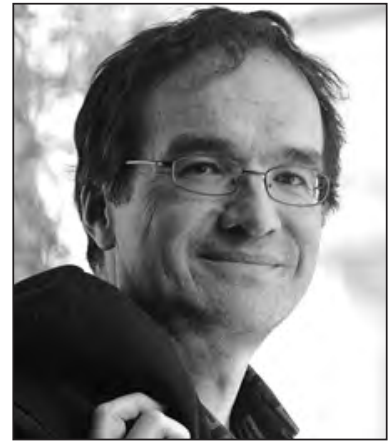

Alain Olivier

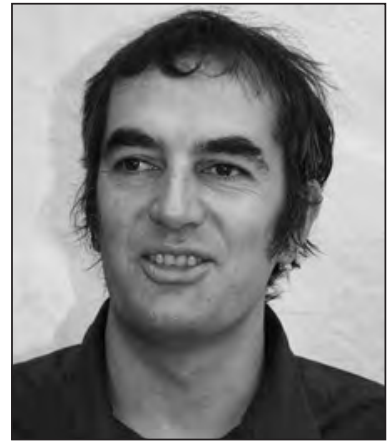

Fabien Liagre

\section{Introduction}

En Amérique du Nord comme en Europe, au cours du siècle dernier, les systèmes agricoles et sylvicoles ont connu une importante spécialisation et une forte intensification qui ont permis d'augmenter considérablement les productions. Cependant, les conséquences écologiques qui en ont découlé remettent aujourd'hui en cause la durabilité de ces systèmes. Depuis une trentaine d’années, de nombreuses études ainsi qu'une expérience de terrain grandissante ont montré que la présence d’arbres sur le territoire agricole permet de générer plusieurs services écosystémiques et bénéfices environnementaux comme la séquestration du carbone, la conservation de la biodiversité et lamélioration de la qualité de l'eau, du sol et des paysages (Palma et al. 2007, Jose 2009, Quinkenstein et al. 2009). Les arbres hors forêts ont également un intérêt économique pour les populations rurales (FAO 2001). Le maintien et la restauration d'arbres hors forêts contribuent ainsi à rendre possible la pratique d'une agriculture durable. De plus, la polyvalence de l'arbre en fait un outil qui coïncide bien avec les enjeux de l'agriculture multifonctionnelle d'aujourd'hui (Nihous 2008). En effet, l'agriculture est aujourd'hui reconnue comme assumant un rôle essentiel dans la structuration du territoire et le modelage des paysages, autant que dans la garantie d'une production de denrées alimentaires en quantité suffisante et de qualité (European Conference on Rural Development 1996, Groupe Polanyi 2008).

Les arbres champêtres peuvent être présents sous diverses modalités sur le territoire agricole. Les aménagements peuvent être disposés en alignements en bordure de champshaies et bandes riveraines ligneuses (ripisylves) —ou bien au sein même de parcelles cultivées. Dans ce second type d’aménagement, des lignes d'arbres alternent avec des bandes cultivées ou pâturées dont la largeur est compatible avec le passage de machines agricoles. Un tel système, qui diffère du boisement, permet de mener simultanément une production sylvicole et une production agricole végétale (agrosylviculture ou système de culture intercalaire, Fig. 1) ou animale (sylvopastoralisme). Il s'agit d'une des formes d'association les plus étroites que lon puisse trouver entre arbres et cultures et elle correspond à la définition de l'agroforesterie au sens strict (Somarriba 1992). Dans ces systèmes, les interactions aériennes et souterraines entre les composantes ligneuse et herbacée sont nombreuses. Une bonne gestion permet de favoriser la complémentarité entre les deux composantes et de minimiser la concurrence pour l'approvisionnement en eau, en éléments minéraux et en lumière. L’ensemble de la parcelle profite alors des bénéfices agroenvironnementaux liés aux arbres. Les systèmes de culture intercalaire peuvent de plus générer des revenus diversifiés liés à la production de bois (bois d'œuvre et biomasse) (Graves et al. 2007) tout en réduisant certaines charges (économies en intrants) (Thevathasan et Gordon 2004, Malézieux et al. 2009). Ils permettent ainsi une production intégrée et économiquement rentable.

Les acteurs du développement et de la recherche en agroforesterie en Amérique du nord et en Europe sont conscients de la nécessité d’aborder la production agricole de manière raisonnée et considèrent que les arbres peuvent jouer un rôle fondamental dans cette nouvelle agriculture (Reisner et al. 2007, USDA 2011). Grâce à la mobilisation des acteurs de terrain et au soutien politique et financier des instances gouvernementales, les haies et les bandes riveraines arborées se multiplient. Les systèmes agrosylvicoles et sylvopastoraux quant à eux progressent plus lentement. Les enjeux agricoles et forestiers diffèrent d'un pays à l'autre, impliquant le développement de stratégies différentes vis-à-vis de l'agroforesterie.

En mars 2010, le Comité agroforesterie du Centre de référence en agriculture et agroalimentaire du Québec (CRAAQ) a organisé un forum sur l'agroforesterie. L'objectif était de mobiliser les forces vives en agroforesterie en rassemblant les principaux acteurs (gestionnaires, conseillers, aménagistes du territoire, représentants des producteurs agricoles et forestiers, chercheurs, élus, agents de développement, gens d'affaires) dans les domaines de la recherche, du transfert technologique, de la formation, de la production, de la transformation, de la mise en marché et du financement (CRAAQ 2011). Il en est ressorti de nombreux enjeux, répartis ici en cinq catégories : reconnaissance, interdisciplinarité et approche collective ; acquisition et transfert de connaissances ; statut, réglementations et financement ; sensibilisation et mobilisation ; support technique et mise en œuvre sur le terrain. Dans les pages qui suivent, nous tenterons détablir un parallèle entre la France et le Québec plus particulièrement, en examinant comment chacun de ces enjeux y est abordé. Le texte sarticule selon un va-et-vient entre les conclusions issues du forum québécois, précédées de la men- 


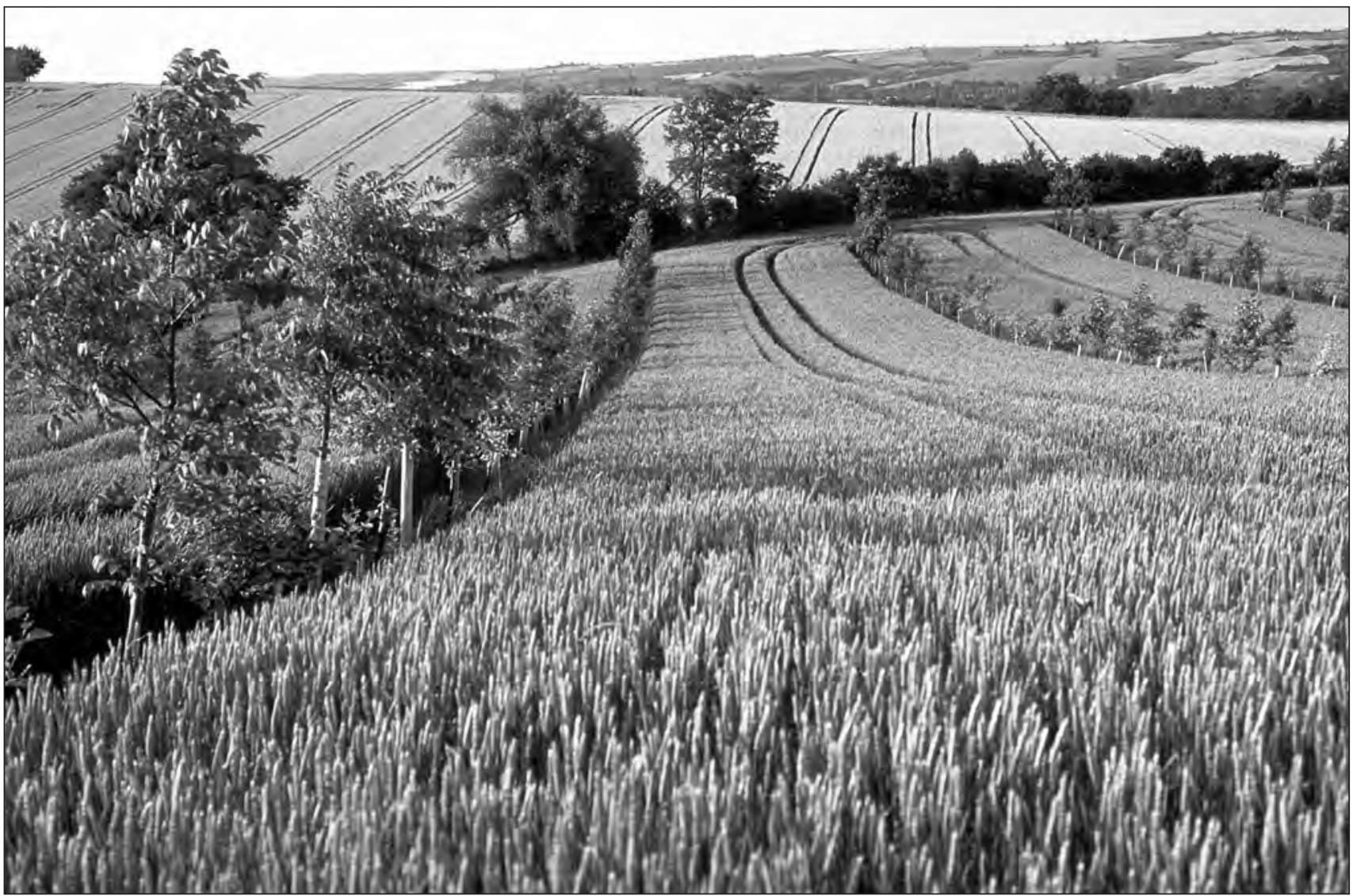

Fig. 1. Jeune système de culture intercalaire, illustrant les principes de l'agroforesterie de seconde génération, Gers, France (photo Raymond Sauvaire].

tion $[\mathrm{QC}]$, et les propos se rapportant à la situation française, précédés de la mention [FR]. De l’analyse de la situation de lagroforesterie dans ces deux territoires, se dégagent plusieurs éléments également valables dans d’autres régions agricoles canadiennes analogues comme l'Ontario et les Maritimes.

\section{Reconnaissance, interdisciplinarité et approche col- lective}

\section{Reconnaissance}

[QC] Le forum a fait ressortir un manque de vision collective. Or, un soutien politique est indispensable au développement de pratiques innovantes. Des initiatives du ministère de l'Agriculture, des Pêcheries et de l'Alimentation du Québec (MAPAQ), comme le programme de conservation des sols et de l'eau et le programme Prime-Vert, ont par exemple encouragé l'établissement de haies brise-vent, contribuant à en faire le principal système agroforestier au Québec aujourd'hui. Les bandes riveraines arborées suscitent elles aussi de plus en plus d'intérêt, notamment en raison de leur contribution à l'amélioration de la qualité de l'eau. Cependant, à l'heure actuelle, la contribution potentielle de larbre hors forêt à de grands enjeux comme le changement climatique ou la biodiversité ne semble pas évidente pour tous, et ne fait pas lobjet de soutien financier à long terme. Une meilleure connaissance de cette contribution par les décideurs politiques permettrait sans doute d’augmenter la reconnaissance de lagroforesterie au plan politique.
[FR] Au cours des dernières décennies, la politique agricole commune (PAC), qui oriente, réglemente et finance l'agriculture européenne, a, tout comme Agriculture et Agroalimentaire Canada (AAC 2011), étendu ses préoccupations au-delà du seul aspect économique, en intégrant de plus en plus de mesures en faveur de la préservation de lenvironnement. Ainsi, lattribution des aides agricoles est maintenant soumise à une éco-conditionnalité (respect de sept «bonnes conditions agroenvironnementales »), comme c'est aussi le cas au Québec (présentation d'un bilan phosphore et d'un plan agroenvironnemental de fertilisation conformes) (Règlement sur l'enregistrement des exploitations agricoles et sur le paiement des taxes foncières et des compensations 2011). La PAC se divise en deux " piliers » : le premier pilier concerne les aides à la production agricole, tandis que le second pilier, appelé Règlement de développement rural (RDR), constitue un cadre financier et un programme de développement rural unique pour toute l'Union européenne. De nombreuses mesures agroenvironnementales (MAE) sont financées dans le cadre de ce second pilier, de même quau Québec, le programme Prime-Vert soutient la diffusion des « bonnes pratiques agroenvironnementales » (MAPAQ 2005). En effet, il a été reconnu que, loin de nuire à la production, ladoption de pratiques culturales favorisant la protection des eaux et des sols et la biodiversité permet d'envisager une agriculture à la fois productive et durable (Le Roux et al. 2008). Dans ce contexte de « verdissement » de l'agriculture, le RDR en Europe, 
tout comme Prime-Vert au Québec, soutient depuis quelques années l'aménagement de haies et de bandes riveraines. Un important travail de recherche, de démonstration et de communication sur le thème de l'agroforesterie a été réalisé par l'Institut national de la recherche agronomique (INRA), notamment au cours du projet de recherche agroforestier européen SAFE (Silvoarable Agroforestry For Europe ; Systèmes Agroforestiers pour les Fermes Européennes) conduit entre 2001 et 2005. Ce travail a abouti à la reconnaissance du rôle de l'arbre hors forêt dans la réponse aux enjeux agricoles, mais aussi écologiques, climatiques et énergétiques en Europe (Dupraz et al. 2005). Pour la période 2007 à 2013, la PAC comporte ainsi une mesure spécifique aux systèmes de culture intercalaire, ce qui constitue une avancée décisive pour l'agroforesterie européenne, en rendant l'agrosylviculture compatible avec les réglementations et la juridiction agricoles.

Preuve de sa transversalité, l'agroforesterie au sens large, c'est-à-dire l'ensemble des systèmes de production associant arbres et cultures, contribue à rencontrer les objectifs fixés par plusieurs textes cadre européens : plan climat de l'Union européenne, stratégie européenne pour la biodiversité, directive cadre sur l'eau. En France, la loi Grenelle II de 2010, loi portant sur les engagements nationaux pour l'environnement, a inséré comme objectif dans les codes de l'environnement et de l'urbanisme la formation de maillages arborés et a fait de la « trame verte et bleue » une mesure phare. La France a par ailleurs rendu éligibles les parcelles agroforestières aux aides agricoles du premier pilier de la PAC pour des densités inférieures à 200 arbres/ha. Cette disposition est pionnière en Europe, où la plupart des pays ont conservé une densité maximale de 50 arbres/ha.

\section{Interdisciplinarité}

[QC] La séparation entre le domaine de l'agriculture et celui de la foresterie est marquée dès le niveau ministériel, puisque les activités agricoles relèvent du MAPAQ, tandis que les activités sylvicoles se rattachent au ministère des Ressources naturelles et de la Faune (MRNF), ce qui constitue un frein à la reconnaissance de l'agroforesterie. En raison de la transversalité des impacts de l'arbre hors forêt, les participants du forum encouragent la constitution de comités interdisciplinaires comme ceux du CRAAQ et les approches multidisciplinaires.

[FR] De même, l'Association française des arbres et haies champêtres (AFAHC) souligne que « le développement que connaît aujourd'hui l'arbre champêtre est le fruit d'une forte implication des opérateurs locaux et des planteurs volontaires, mais c'est aussi le fait de différents acteurs de la société qui s'y sont investis : chercheurs, agents de développement, socioprofessionnels, agents administratifs, etc. qui collaborent localement ou participent directement aux deux structures nationales, l'Association française des arbres et haies champêtres et l'Association française d'agroforesterie (AFAF) ».

\section{Approche collective}

[QC] Les participants du forum ont insisté sur la nécessité de mettre en place une stratégie québécoise de développement de l'agroforesterie dans la province. Selon eux, il faudrait insérer l'agroforesterie dans les plans régionaux de développement et identifier en régions les zones prioritaires pour les aménagements arborés, en concertation avec les principaux intervenants du milieu. Grâce à son rôle de concertation, le CRAAQ semble à même de jouer le rôle d'interface entre les Ministères et les intervenants locaux ainsi que de sensibiliser le milieu municipal.

[FR] En effet, les enjeux de l'arbre dépassent le cadre de l'exploitation agricole : c'est tous les acteurs du territoire qui sont concernés. Au lieu de les opposer, l'arbre peut faire converger les objectifs des différents acteurs : l'agriculteur constitue un capital foncier, améliore et diversifie sa production, les collectivités territoriales sécurisent leurs routes et embellissent leur territoire, les structures de gestion de l'eau contribuent à la protection des nappes, des cours d'eau et des berges, les écologues favorisent la préservation voire la restauration de la diversité végétale et animale, les chasseurs bénéficient d'habitats favorables au petit gibier, etc. En France comme au Québec, l'a priori selon lequel production et préservation sont incompatibles fait encore obstacle à la revalorisation des ligneux en milieu agricole. Mais, en France, certains Conseils régionaux, Conseils généraux ou Agences de l'eau contribuent financièrement à la restauration déléments arbustifs et arborés sur des terres agricoles. Ainsi, le Conseil régional de Midi-Pyrénées appuie financièrement la plantation de haies grâce à la mise en place du Fonds Régional Carbone, qui finance des actions contribuant à réduire l'empreinte écologique de la région. En 2011, les deux tiers des régions françaises ont adopté la mesure 222 du RDR européen qui vise à soutenir l'investissement en agroforesterie. Larbre devient alors un outil efficace d'aménagement durable du territoire et un beau symbole de conciliation entre économie et écologie.

\section{Acquisition et transfert de connaissances Acquisition de connaissances}

[QC] Comme cela a été dit précédemment, les haies brisevent et, plus récemment, les bandes riveraines arborées constituent les principales formes agroforestières de la province. Cependant, des expérimentations sont encore nécessaires pour évaluer leur efficacité dans le contexte biophysique du Québec, notamment en fonction de leur configuration, de leur composition, de leur connectivité, etc. Pour leur part, les systèmes agrosylvicoles ont fait l'objet de plusieurs études menées dans l'Est du Canada, qui ont démontré leur potentiel de production et leur capacité à répondre à divers enjeux environnementaux (Bradley et al. 2008, Rivest et al. 2010). Dans le cadre du Programme de lutte contre les gaz à effet de serre en agriculture d'AAC, un groupe de chercheurs canadiens a amorcé en 2011 (à 2014) une étude visant à déterminer le potentiel des systèmes de cultures intercalaires à atténuer les émissions de gaz à effet de serre et à séquestrer le carbone dans la biomasse végétale et dans les sols agricoles. La contribution potentielle des systèmes de cultures intercalaires à l'adaptation aux changements climatiques anticipés attire aussi l'attention d'une équipe de chercheurs québécois dans le cadre d'un programme du Consortium sur la climatologie régionale et l'adaptation aux changements climatiques (Ouranos 2011-2013). Les résultats de ces études permettront notamment d'orienter les pouvoirs publics dans l'adoption éventuelle de mesures permettant d'atténuer les effets des changements climatiques anticipés et de s'y adapter en agriculture. 
[FR] L’année 2005 a vu l'aboutissement du vaste projet de recherche agroforestier SAFE. Les études menées dans les divers pays d'Europe avaient alors conclu à la pertinence agronomique, écologique, économique et sociale des aménagements agroforestiers et à la compatibilité de l'arbre avec les agrosystèmes modernes (Dupraz et al. 2005). Depuis, d’autres projets se sont succédés dans le cadre du programme national de développement agricole et rural. Le programme CASDAR (Compte d'Affectation Spéciale Développement Agricole et Rural) 2006-2008 a ainsi permis de fédérer les acteurs de la recherche-développement dans une association nationale, faire le bilan des projets expérimentaux, travailler sur des outils d'aide à la décision, et mettre en place un réseau de parcelles de référence (Liagre 2008). Actuellement en cours, le programme CASDAR 2009-2011 a pour objectifs de mesurer et améliorer l'efficacité agro-écologique des systèmes agroforestiers (AFAF 2009).

$\mathrm{Au}$ cours de ces années de recherche, la conception de l'agroforesterie en France a évolué vers une agroforesterie dite de deuxième génération, plus durable et diversifiée. Le raisonnement des aménagements passe par la compréhension des reliefs, sols et climats dans lesquels s'insère lexploitation, ainsi que par l'observation des structures paysagères traditionnelles. Les éléments clefs sont l'emploi de plants d’origine locale, la diversité des essences au sein des aménagements et la variété des formes. Pour la restauration de haies et de bandes riveraines, la gestion de la végétation spontanée est privilégiée, la plantation nétant effectuée qu’en dernier recours. Dans les systèmes de culture intercalaire, la diversité inter et intra spécifique est favorisée par rapport au peuplement uniforme voire monoclonal. D'autre part, les bandes au pied des arbres ne sont plus désherbées mais au contraire végétalisées, ce qui renforce leur action filtrante et antiérosive et leur contribution à la biodiversité. Une attention particulière est portée à la connectivité des divers éléments entre eux (Labant 2009).

De même qu'au Canada (Nolet et al. 2011), un important travail est à présent en cours sur la quantification des biens et services environnementaux fournis par les arbres champêtres, notamment en termes de stockage de carbone. Le Centre de coopération internationale en recherche agronomique et développement (CIRAD), l'INRA de Montpellier et l'École nationale supérieure d'agronomie de Montpellier (ENSAM), notamment, travaillent en coopération au sein d'une unité mixte de recherche (UMR System). Ces organisations de recherche disposent pour cela dans le sud de la France du plus ancien site expérimental d'agroforesterie en Europe. Il est depuis 1994 le support d'activités de recherche exclusivement tournées vers les systèmes de culture intercalaire, et une douzaine déquipes y travaillent actuellement dans le cadre du programme intégré de recherches en agroforesterie à Restinclières (PIRAT). De nombreux projets pilotes sappuyant sur le réseau de parcelles mis en place sont également menés.

Létablissement d'un réseau de parcelles de référence est une étape clef pour juger de la faisabilité des diverses pratiques agroforestières dans les conditions locales. Au Québec, la grande variabilité des conditions climatiques et biophysiques et des enjeux d'une région à l'autre rend cette étape décisive dans un objectif de généralisation de l'agroforesterie à léchelle de la province. La multiplication des échanges entre la France et le Québec est également une source d’acquisition de connaissances pour les deux parties à ne pas négliger.

\section{Transfert de connaissances}

[QC] Lors du forum, un manque de transfert des connaissances a été déploré. Les participants suggèrent de mandater un organisme pour compiler les résultats de recherche et l'expertise terrain. Le CRAAQ pourrait jouer un rôle dans la diffusion des connaissances à grande échelle. En régions, un transfert efficace de connaissances passe par la mise en place d'un réseau de professionnels qualifiés en agroforesterie. Létablissement d'un réseau de parcelles de démonstration est également primordial, notamment en ce qui concerne les systèmes agrosylvicoles innovants. Notons qu'à Québec, l'Université Laval propose le seul programme de formation universitaire francophone en agroforesterie.

[FR] Pour la revalorisation de l'arbre hors forêt à léchelle nationale, la proximité entre acteurs et décideurs est primordiale. En France, ce rôle d'interface est assuré par deux structures nationales complémentaires : l'AFAF - qui assume depuis 2007 un rôle de référence concernant l'agrosylviculture et le sylvopastoralisme - et l'AFAHC - qui fait le pendant en ce qui concerne les aménagements agroforestiers de bordure de parcelle, de rivière ou de route, tels que les haies ou les bandes riveraines. Des associations locales de revalorisation de larbre champêtre, comme Arbre \& Paysage 32, assurent également la transmission des connaissances acquises grâce à la recherche ainsi que des savoir-faire développés avec l'expérience du terrain depuis maintenant une trentaine d’années. Concrètement, cela passe par la réalisation de documents cadre, lorganisation de visites et de formations à destination des agriculteurs, professionnels de lagriculture et du paysage, élus, agents des collectivités, services de l'État ou autre, lorganisation de voyages d'études, l'intervention dans les établissements de formation professionnelle, scolaire et universitaire, etc. D’autre part, Agroof, bureau détude spécialisé en agroforesterie, collabore fréquemment avec l'INRA sur des projets de recherche et assume parallèlement un rôle de formation professionnelle et de vulgarisation, ce qui contribue au transfert efficace des connaissances. L'agroforesterie est à ce jour absente des programmes de formations agricole et agronomique, mais un premier mouvement se dessine : depuis 2011, quelques structures d'enseignement envisagent en effet d'intégrer lagroforesterie dans leur tronc commun denseignement (Institut polytechnique Lasalle de Beauvais et École supérieure d'ingénieurs et de techniciens pour lagriculture de Rouen).

\section{Statut, règlementations et financement Statut}

[QC] Larbre agroforestier est un arbre hors forêt, aussi a-t-il eu tendance à être délaissé à la fois par les forestiers, dont la vocation est surtout de gérer des peuplements boisés, et par les professionnels agricoles, qui ne se sentaient pas concernés par lélément ligneux. Forestier ou agricole, quel statut accorder alors à l'arbre agroforestier?

Au Québec, le statut de l'agroforesterie est encore flou mais les arbres, même hors forêt, demeurent liés à la foresterie, comme en témoigne l'implication d'intervenants de terrain et de chercheurs issus du milieu forestier. Il en est de même de la récolte, voire la culture, dans des boisés, de produits forestiers non ligneux (PFNL) destinés à différents marchés (alimentaire, pharmaceutique, cosmétique, ornemental), une pratique agroforestière qui suscite de plus en plus d'intérêt au Québec. En effet, certains agriculteurs ou propriétaires ter- 
riens possèdent un bois inexploité et souhaiteraient le mettre en valeur. Aussi la culture sous couvert est-elle une voie de développement prometteuse pour l'agroforesterie au Québec (De Baets et al. 2007). D'autre part, suite à la démonstration des bons résultats de croissance des arbres agroforestiers (Rivest et al. 2009) et de la possibilité de produire des arbres de haute valeur commerciale en milieu agricole, l'intérêt de l'agroforesterie pour la production de bois est manifeste. Les participants du forum comptent donc sur le soutien financier du secteur forestier.

[FR] En France, suite au programme CAS DAR, l'agroforesterie a été résolument rattachée au secteur agricole. La circulaire ministérielle du 20 avril 2010 spécifie que le statut agroforestier relève bien du statut agricole. Sur les exploitations, l'arbre est désormais considéré comme une composante à part entière de l'agrosystème (APCA 2010).

\section{Réglementations}

[QC] À ce jour, les systèmes agroforestiers québécois ne font lobjet d'aucune réglementation spécifique. Pour formaliser ces aménagements, il apparaît nécessaire détablir des seuils de référence. Par exemple, quelle est la largeur valable d'un système riverain agroforestier ? Dans le cas des systèmes de culture intercalaire ou du sylvopastoralisme, les arbres ne sont pas juxtaposés aux cultures mais superposés, ce qui tend à compliquer la mise en place de réglementations appropriées. Un contexte législatif complexe présentant des lois et politiques conflictuelles, particulièrement en matière de boisement de terres en friche en zone agricole, restreint ainsi pour le moment les systèmes agrosylvicoles à des projets pilotes (De Baets et al. 2007, Marchand et Masse 2007). De plus, les surfaces utilisées pour la plantation de lignes d'arbres ne bénéficient pas du remboursement de la taxe foncière, contrairement aux surfaces en culture.

[FR] En Europe, la largeur des haies et bandes riveraines arbustives éligibles aux aides du premier pilier de la PAC varie d'un département à l'autre mais est généralement de l'ordre de $4 \mathrm{~m}$. Le cas des systèmes de culture intercalaire a été pris en compte plus récemment. Avant 2001, une parcelle agroforestière nétait pas éligible aux aides agricoles. En 2001, la France a accepté le principe de l'éligibilité des surfaces intercalaires en agroforesterie. En 2006, cette disposition s'est élargie aux parcelles de moins de 50 arbres/ha dans leur totalité, surface occupée par les arbres comprise. Enfin, en 2010, cette densité est passée à 200 arbres/ha. La réalisation de nombreux projets avait déjà montré que l'arbre était compatible avec les systèmes agricoles sur les plans biologique et économique, il l'est à présent également sur le plan juridique. Cela a permis aux systèmes agrosylvicoles et sylvopastoraux de se développer en France à travers des initiatives individuelles de plus en plus nombreuses. Jusqu’ici, de même qu’au Québec, la mobilisation des aides pour ces systèmes était soumise à des critères contraignants et impliquait des démarches administratives complexes qui avaient de quoi décourager les planteurs.

Dans le cadre du verdissement de la PAC, les aides européennes à la production sont conditionnées par le respect de sept « bonnes conditions agroenvironnementales » (BCAE). Depuis 2010, une nouvelle BCAE impose le maintien de particularités topographiques sur $3 \%$ de la surface agricole utile de l'exploitation. Ces particularités topographiques peuvent être des cours d'eau, des mares, des jachères, des murets... ou des arbres. Les aménagements agroforestiers sont alors convertis en surfaces équivalentes topographiques (SET). Par exemple, $1 \mathrm{~m}$ linéaire d'alignement d'arbres en plein champ correspond à $10 \mathrm{~m}^{2}$ de SET et 1 ha de verger pâturé correspond à 5 ha de SET. Ce seuil de $3 \%$ en surfaces non travaillées, ni fertilisées, ni traitées est déjà atteint sur de nombreuses exploitations, excepté dans des régions fortement céréalières comme la Beauce. Pour les producteurs de ces régions, l'implantation d'arbres sur l'exploitation peut être un moyen de respecter la réglementation tout en bénéficiant d'avantages agroenvironnementaux.

\section{Financement}

[QC] Aujourd'hui, le volet 10 du programme Prime-Vert subventionne l'implantation de haies brise-vent et de bandes riveraines arborées jusquà $90 \%$ des coûts admissibles. Cependant, il n'existe actuellement aucun programme d'aide pour l'entretien des aménagements agroforestiers, ce qui remet en cause la viabilité des projets sur le long terme ainsi que leur potentiel de production (bois d'œuvre, biomasse, PFNL).

[FR] En France, une mesure dans le cadre du Plan de développement rural hexagonal (PDRH) permet de bénéficier d'une aide à l'entretien des haies, des arbres isolés ou en alignements, des ripisylves et des bosquets. Le bénéficiaire s'engage alors pour une durée de cinq ans, au cours desquels des visites de contrôles peuvent être effectuées.

[QC] Il n'existe à ce jour aucun système d'aide spécifique aux systèmes de culture intercalaire et au sylvopastoralisme au Québec. Il n'y a pas de porteur de dossier clairement identifié au gouvernement provincial pour l'agrosylviculture, ce qui complique les demandes de financements. Les objectifs de travail qui sont ressortis lors du forum sont : « la création d'un programme d'aide spécifique à l'agroforesterie conjoint entre le MAPAQ et le MRNF et l'assouplissement des programmes existants de façon à rendre le financement plus accessible et flexible aux différents contextes sur le terrain ».

En 2011, le MAPAQ a toutefois lancé le programme pilote d'appui à la multifonctionnalité de l'agriculture. Pour la première fois au Québec, les projets individuels ou collectifs visant la mise en place de pratiques agroforestières émergeantes comme les systèmes de culture intercalaire peuvent être soutenus financièrement par l'État. Spécifiquement, ce programme vise à soutenir l'adoption, par les entreprises agricoles, de pratiques qui contribuent à la mise en valeur des paysages, à l'attractivité des territoires, à l'accessibilité de l'espace rural, à la préservation du patrimoine agricole, à la protection de la biodiversité et la prestation de services destinés à la communauté. Cependant, seules certaines régions sont concernées par ce programme, en particulier celles dont le potentiel agricole est limité. Plusieurs des régions où se pratique une agriculture intensive, comme la Montérégie et le Centre-du-Québec, ne sont pas admissibles à ce programme. En outre, seules les entreprises agricoles à revenus faibles à modérés y sont éligibles.

[FR] En 2001, la France a mis en œuvre dans son PDRH une mesure de soutien à l'investissement agroforestier. Cette initiative a inspiré le RDR européen pour la période 2007 à 2013, qui inclut à son tour une mesure de soutien (mesure 222). Dans un premier temps et de manière très surprenante, la France n'a pas adopté dès 2007 cette mesure dans le cadre 
du plan national pour le développement rural (PNDR), déclinaison nationale du RDR. Le travail de démonstration et de concertation des acteurs du développement agroforestier français a finalement abouti en 2010 à louverture de cette mesure dans le PNDR. Ainsi, dans toutes les régions ayant activé la mesure 222, les plantations intraparcellaires peuvent désormais prétendre à un soutien de l'Union européenne allant jusquà $70 \%$ des coûts admissibles. Lagriculteur s'engage alors à entretenir l'aménagement ayant fait lobjet de laide pour une durée de cinq ans. Les travaux pris en compte sont la conception du projet, lélimination de la végétation préexistante, la préparation du sol, la fourniture, la mise en place, la protection et le paillage des plants forestiers, et le suivi technique de la plantation pendant trois ans. Des structures locales de gestion du territoire peuvent également se porter volontaires pour participer financièrement à ces projets. Plusieurs conditions fondamentales sont nécessaires pour être éligible à cette aide, parmi lesquelles : (i) la densité des arbres doit être comprise entre 30 et 200 arbres/ha et être compatible avec l'activité agricole ; (ii) les activités agricoles et sylvicoles doivent être menées simultanément sur la même parcelle; (iii) les arbres forestiers choisis doivent respecter la règlementation régionale relative aux matériels forestiers de reproduction concernant les provenances d'espèces forestières adaptées aux conditions climatiques locales; (iv) les paillages utilisés doivent être biodégradables ; (v) les produits phytosanitaires sont interdits sur les lignes végétalisées après la deuxième année.

\section{Sensibilisation et mobilisation}

[QC] Les acteurs de l'agroforesterie québécoise notent un intérêt grandissant des agriculteurs pour l'arbre mais soulignent le besoin détudes sociales pour comprendre la relation qu'ils entretiennent avec celui-ci et leurs motivations à implanter des systèmes agroforestiers.

[FR] En Europe, une enquête réalisée dans sept pays auprès de 264 producteurs agricoles avait révélé qu'en 200550 $\%$ d'entre eux se disaient prêts à faire l'essai de pratiques agroforestières (Graves et al. 2009). Aujourd'hui de plus en plus d’agriculteurs français sont intéressés par l'idée de réintroduire larbre dans leur système dexploitation (AFAF 2010, communication personnelle). Il est intéressant de constater que cette tendance n'est pas imposée par les politiques agricoles mais que c'est au contraire l'impulsion émanant du terrain (producteurs, associations, chercheurs) qui fait évoluer les réglementations. L'AFAF et l'AFAHC interviennent ainsi auprès des instances ministérielles et participent aux différentes consultations aux niveaux national, régional et local, afin d'aboutir à une meilleure reconnaissance officielle. Ces deux associations contribuent également, avec le concours d'intervenants locaux, à la diffusion de l'information à large échelle, à travers des sites internet, la publication douvrages, livrets, périodiques et documentaires, lorganisation dévénements et de conférences, etc. Au Québec, ce rôle est joué, dans une certaine mesure, par le comité agroforesterie du CRAAQ, qui s'implique dans la promotion de pratiques agroforestières innovantes. Ce comité est né de la concertation de leaders en agroforesterie issus de divers milieux : agriculture, forêt, environnement, aménagement du territoire rural. Il est composé d'une équipe multidisciplinaire de professionnels (praticiens, aménagistes, chercheurs, gestionnaires) œuvrant dans diverses régions du Québec. À court terme, le Comité agroforesterie du CRAAQ souhaite initier un réseau structuré de sites de démonstration, établir un répertoire des intervenants en agroforesterie et organiser un nouvel événement technique et scientifique en agroforesterie. L'Union des producteurs agricoles (UPA), les clubs-conseils en agroenvironnement (CCAE), la Coop fédérée, la Financière agricole, les agences forestières et les groupements forestiers sont d'autres acteurs qui pourraient également prendre part au développement de l'agroforesterie au Québec.

[QC] La mise en œuvre cohérente sur le terrain passe également par une implication accrue des collectivités territoriales au niveau local. Il est donc essentiel de sensibiliser les élus et employés municipaux, en adaptant les pratiques et le discours en fonction des enjeux et problématiques de chaque région.

[FR] En France, certaines chambres d'agriculture proposent un accompagnement technique aux planteurs et certains Conseils régionaux participent financièrement aux projets de plantation dans leur région. Au Québec, les Conférences régionales des élus (CRE) ou les Municipalités régionales de comtés (MRC) pourraient de même appuyer la revalorisation de larbre sur leur territoire. Pour les régions agricoles confrontées à une raréfaction des milieux forestiers, l'agroforesterie pourrait être un élément de solution à intégrer dans les actions du Plan régional de développement intégré des ressources naturelles et du territoire (PRDIRT) en cours délaboration pour le compte du MRNF.

\section{Support technique et mise en œuvre sur le terrain}

[QC] La réussite d'un projet agroforestier est conditionnée par le respect de quelques principes fondamentaux lors de la plantation. Laccompagnement technique au moment de la conception du projet, de la plantation et des premières années de croissance de larbre est donc fondamental. Au cours du forum, un manque dexpertise technique été déploré par les participants. Le souhait a été émis quà brève échéance au moins un expert en agroforesterie soit disponible dans chaque région, par exemple à la direction régionale du MAPAQ ou dans un CCAE. Le support technique pour l'entretien des arbres pourrait être apporté aux producteurs par les servicesconseils en foresterie coordonnés par les Agences forestières et réalisé par les conseillers forestiers, syndicats de producteurs de bois, coopératives ou groupements forestiers.

[FR] En France, la mise en ouvre technique et administrative des projets agroforestiers sest simplifiée au fil des ans, les rendant moins contraignants. Grâce aux aides européennes ils sont désormais relativement peu coûteux à implanter. Dans la plupart des départements se trouvent des personnes ressources qui disposent de la compétence et de l'expertise de terrain locale pour accompagner les planteurs. Pendant les premières années qui suivent limplantation, cet accompagnement permet de réduire le taux de mortalité à $3 \%$ à $4 \%$ (AFAF 2010, communication personnelle). Le réseau d'experts est cependant encore à renforcer.

[QC] La question de lapprovisionnement en plants forestiers de qualité constitue également un élément important. Au Québec, il est parfois difficile de se procurer des espèces d'arbres feuillus à bois noble. Actuellement, des plants sont fournis par le MRNF, mais certaines espèces d'arbre et les arbustes ne sont pas proposés. Des pépinières locales pour- 
raient être développées et loffre (quantité et diversité) du MRNF pourrait être accrue, pour assurer un approvisionnement constant en plants de qualité d'espèces nobles et indigènes. Les participants au forum ont insisté sur l'importance de miser sur la conservation et la reproduction d'espèces indigènes.

[FR] En France, depuis peu, chaque région définit une liste des espèces autorisées pour la plantation en agroforesterie. Il sagit souvent despèces indigènes, dont les plants sont issus d'une sélection locale, en accord avec les principes de base de l'agroforesterie de deuxième génération.

Enfin il convient de souligner que, pour répondre efficacement aux enjeux environnementaux des exploitations, lagroforesterie est indissociable des bonnes pratiques agricoles, comme la réduction de l'utilisation d'intrants et la diminution du travail du sol.

\section{Conclusion}

De manière générale, l'analyse du développement récent de l'agroforesterie au Québec et en France fait ressortir l'importance de l'approche par territoire. En effet, au-delà de la pertinence économique et environnementale de l'agroforesterie dans le système d'exploitation, les arbres hors forêt assument un rôle plus large à léchelle du territoire, notamment par la production dénergie renouvelable, le stockage de carbone et lembellissement du paysage. Dans les régions de grandes cultures où l'agriculture est pratiquée de manière intensive, les systèmes agroforestiers peuvent être intéressants pour leur aptitude à répondre à une urgence environnementale en atténuant la pollution diffuse et la perte des sols, et en accroissant la biodiversité. Dans des zones où l'agriculture est devenue plus marginale et où la déprise est en cours, ils peuvent, grâce à leurs fonctions productive et paysagère, constituer une alternative au reboisement en rentabilisant des terres à faible potentiel et en créant un cadre de vie attractif. Les régions ont tendance à gagner en autonomie. Cela peut être loccasion pour les pouvoirs locaux de se saisir de loutil multifonctionnel quest larbre pour valoriser leurs ressources de manière durable.

En France, la reconnaissance politique de l'agroforesterie est acquise, et les acteurs du développement constatent une augmentation du nombre d'initiatives agroforestières de la part des professionnels de l'agriculture et de la gestion du territoire. Les enjeux consistent désormais à faire prendre conscience aux multiples acteurs ruraux du formidable outil de conciliation que constitue l'arbre. Des efforts sont également fournis pour accompagner individuellement les planteurs et garantir la mise en œuvre de projets localement adaptés et cohérents. Cela passe par létablissement sur l'ensemble du territoire d'un réseau d'interlocuteurs locaux disponibles et qualifiés.

Larbre champêtre a sa place dans les paysages ruraux et dans les agrosystèmes. Cependant, il est probable qu’au préalable le statut de larbre hors forêt devra être clarifié. Cest notamment crucial dans les secteurs d'agriculture intensive du Québec, de l'Ontario et des Maritimes où un certain nombre de freins juridiques et réglementaires devront être levés. D’autre part, des projets de recherche-développement sont encore nécessaires pour concevoir des aménagements diversifiés et adaptés localement. Quant à la concrétisation sur le terrain, elle nécessitera la mobilisation dopérateurs agricoles et forestiers mais aussi des gestionnaires du territoire et des instances municipales, et la mise en place d'un accompagnement technique efficace, notamment en ce qui concerne l'entretien des aménagements.

La France a résolument identifié l'agroforesterie comme un système de production agricole, tandis que dans l'est du Canada l'arbre semble plus difficilement dissociable du monde forestier. En ce qui a trait à la réglementation, au financement ou à l'accompagnement technique, cela implique une organisation complètement différente de celle retrouvée en France. Cependant, le parallèle effectué ici révèle que certaines notions sont généralisables. Ainsi, l'évaluation de l'efficacité économique et environnementale des divers systèmes agroforestiers, létablissement d'un réseau de parcelles de démonstration, le travail en concertation, ladaptation des stratégies et des pratiques aux contraintes biophysiques et aux enjeux de chaque région, l'accompagnement technique lors de la plantation et des premières années d'entretien, etc., sont autant de points clefs sur lesquels nous avons tout à gagner à partager nos expériences avec celles de la France.

\section{Références}

[AAC] Agriculture et Agroalimentaire Canada. 2011. Stratégie de développement durable du Ministère. Complément du Rapport sur les plans et les priorités 2011-2012 d'Agriculture et Agroalimentaire Canada. En ligne : http://www4.agr.gc.ca/AAFC-AAC/displayafficher.do?id=1299623763856\&lang=fra [Consulté le 30 mai 2011]. [APCA] Assemblée Permanente des Chambres d'agriculture. 2010. Lagroforesterie dans les réglementations agricoles. APCA, Ministère de l'Agriculture, Paris. $17 \mathrm{p}$.

[AFAF] Association française d'agroforesterie. 2009. CAS DAR 2009/2011. En ligne : http://www.agroforesterie.fr/CASDAR/ 20092011/casdar0911.html [Consulté le 20 avril 2011].

Bradley, R.L., A. Olivier, N. Thevathasan et J. Whalen. 2008. Environmental and economic benefits of tree-based intercropping systems. Policy Options, February 2008: 46-50.

[CRAAQ] Centre de référence en agriculture et agroalimentaire du Québec. 2011. Compte-rendu du forum organisé par le Comité agroforesterie du Centre de référence en agriculture et agroalimentaire du Québec à Québec le 3 mars 2010. En ligne : http:// www.craaq.qc.ca/Documents/Evenements/AGROFORESTERIE/C R_AGF_forumMars2010_V_Finale.pdf [Consulté le 20 avril 2011]. De Baets N., A. Vézina et S. Gariépy. 2007. Le portrait de l'agroforesterie au Québec. Agriculture et Agroalimentaire Canada, ISBN \# 978-0-662-73573-1. 88 p.

Dupraz, C. et al. 2005. Synthesis of the Silvoarable Agroforestry For Europe project. INRA-UMR System Editions, Montpellier. 254 p. En ligne : http://www.ensam.inra.fr/safe [Consulté le 20 avril 2011].

European Conference on Rural Development. 1996. The Cork Declaration - A living countryside. En ligne : http://ec.europa.eu/ agriculture/rur/cork_en.htm [Consulté le 30 mai 2011].

[FAO] Food and Agriculture Organization of the United Nations. 2001. Les arbres hors-forêt, vers une meilleure prise en compte. Cahier FAO Conservation 35, ISBN \# 92-5-204656-9.

Graves, A.R. et al. 2007. Development and application of bio-economic modelling to compare silvoarable, arable, and forestry systems in three European countries. Ecological Engineering 29: 434-449.

Graves, A.R. et al. 2009. Farmers perceptions of silvoarable systems in seven European countries. Advances in Agroforestry 6: 67-86.

Groupe Polanyi. 2008. La multifonctionnalité de l'agriculture : une dialectique entre marché et identité. Éditions Quae, Paris. 350 p.

Jose, S. 2009. Agroforestry for ecosystem services and environmental benefits: an overview. Agroforestry Systems 76: 1-10.

Labant, P. 2009. Principes d'aménagement et de gestion des systèmes agroforestiers. AFAHC, France. 40 p. 
Le Roux, X. et al. (éditeurs). 2008. Agriculture et biodiversité. Valoriser les synergies. Expertise scientifique collective, rapport, INRA, France. $116 \mathrm{p}$.

Liagre, F. 2008. Mission DAR 2005. Programme agroforesterie 2006-2008. Compte-rendu final du projet. AGROOF, France. 190 p. En ligne : http://www.agroof.net/agroof_dev/documents/casdar 0608/CR_final.pdf [Consulté le 19 mai 2011].

Malézieux, E., Y. Crozat, C. Dupraz, M. Laurans, D. Makowski, H. Ozier-Lafontaine, B. Rapidel, S. de Tourdonnet et M. ValantinMorison. 2009. Mixing plant species in cropping systems: concepts, tools and models. A review. Agronomy for Sustainable Development 29: 43-62.

Marchand, P.P. et S. Masse. 2007. Boisement et agroforesterie en courtes rotations en territoire privé au Québec : examen des lois, règlements, politiques et programmes. Ressources naturelles Canada, Service canadien des forêts, Centre de foresterie des Laurentides, Québec, ISBN \# 978-0-662-07622-3. 63 p.

[MAPAQ] Ministère de l'Agriculture, des Pêcheries et de l'Alimentation. 2005. Bonnes pratiques agroenvironnementales pour votre entreprise agricole. Deuxième édition. MAPAQ, Québec. 43 p.

Nihous, F. 2008. La diversification et la valorisation des activités agricoles au travers des services participant au développement rural. Rapport de mission présenté au ministère de l'Agriculture et de la Pêche, France. 52 p. En ligne : http://agriculture.gouv.fr/la-diversification-et-la [Consulté le 25 mai 2011].

Nolet, J., M. Mondon et S. Zussy. 2011. Biens et services écologiques et agroforesterie : l'intérêt du producteur agricole et de la société. Agriculture et Agroalimentaire Canada, ISBN \# 978-1-1007154-4. $42 \mathrm{p}$.
Palma, J.H.N. et al. 2007. Modeling environmental benefits of silvoarable agroforestry in Europe. Agriculture, Ecosystems and Environment 119: 320-334.

Quinkenstein, A., J. Wöllecke, C. Böhm, H. Grünewald, D. Freese, B.U. Schneider et R.F. Hüttl. 2009. Ecological benefits of the alley cropping agroforestry system in sensitive regions of Europe. Environmental Science and Policy 12: 1112-1121.

Reisner, Y., R. de Filippi, F. Herzog et J. Palma. 2007. Target regions for silvoarable agroforestry in Europe. Ecological Engineering 29: 401-418.

Rivest, D., A. Cogliastro et A. Olivier. 2009. Tree-based intercropping systems increase growth and nutrient status of hybrid poplar: A case study from two Northeastern American experiments. Journal of Environmental Management 91: 432-440.

Rivest, D., A. Olivier et A.M Gordon. 2010. Les systèmes de cultures intercalaires avec arbres feuillus: jumeler production de bois et production agricole tout en protégeant l'environnement. Agriculture et Agroalimentaire Canada, ISBN \# 978-1-100-94279-7. 12 p.

Somarriba, E. 1992. Revisiting the past: an essay on agroforestry definition. Agroforestry Systems 19: 233-240.

Thevathasan, N.V. et A.M. Gordon. 2004. Ecology of tree intercropping systems in the North temperate region: Experiences from southern Ontario, Canada. Agroforestry Systems 61: 257-268.

[USDA] United States Department of Agriculture. 2011. USDA agroforestry strategic framework, fiscal year 2011-2016. USDA, Washington, DC. $35 \mathrm{p}$. 